\title{
Impacto das crises de sibilância em crianças de 0 a 3 anos de uma área de baixa renda no sul do Brasil
}

\author{
Impact of wheezing episodes in children 0 to 3 years old from a low-income area \\ in Southern Brazil
}

\section{Sofia Bezerra de Oliveira Costa ${ }^{1}$, Ana Luiza Tainski Azevedo ${ }^{1}$, Marie Christine Revillion de Oliveira ${ }^{2}$, Leonardo Araujo Pinto ${ }^{3}$}

1 Alunas de Graduação da Faculdade de Medicina da Pontifícia Universidade Católica do Rio Grande do Sul (PUCRS). Porto Alegre, RS

2 Médica Pediatra do Centro de Saúde Bom Jesus. Porto Alegre, RS.

3 Doutor em Saúde da Criança e do Adolescente pela Universidade Estadual de Campinas (UNICAMP). Pesquisador do Centro Infant, Instituto de Pesquisas Biomédicas, PUCRS. Professor do Programa de Pós-graduação em Pediatria e Saúde da Criança da PUCRS. Porto Alegre, RS.

\begin{abstract}
RESUMO
Objetivos: Identificar a prevalência de sibilância e fatores associados em crianças com idade inferior a três anos de uma amostra populacional de baixa renda e medir outros marcadores de gravidade associados à sibilância e à asma nos primeiros anos de vida.

Métodos: Os pais ou responsáveis das crianças incluídas foram entrevistados no centro de saúde de atenção primária de uma região de baixa renda da cidade de Porto Alegre, Rio Grande do Sul, e responderam a um questionário desenvolvido para estimar a prevalência de sibilância e avaliar os fatores associados, denominado EISL (Estudo Internacional de Sibilância em Lactentes). Foram incluídos pacientes com idade até 36 meses que consultavam para puericultura e/ou vacinação. Pacientes trazidos na emergência não foram incluídos.

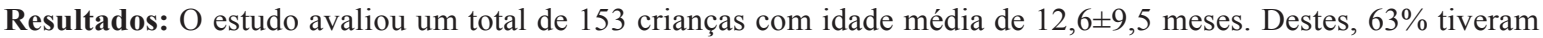
episódios de sibilância relatados, que ocorreram uma ou mais vezes durante os primeiros três anos de vida. A média de idade do primeiro episódio de sibilância foi 4,9 $\pm 5,3$ meses. Utilizaram $\beta 2$-agonistas, corticoides orais e corticoides inalatórios $58,2 \%, 32,7 \%$ e $19 \%$ dos pacientes, respectivamente. Mais de $58 \%$ dos pacientes foram à emergência por sibilância e $13 \%$ foram hospitalizados pelo menos uma vez nos primeiros anos de vida.

Conclusões: Este estudo mostrou que a prevalência de sibilância foi alta entre as crianças pequenas atendidas em um centro de saúde de uma região de baixa renda da cidade de Porto Alegre, sendo que os primeiros sintomas geralmente ocorreram durante os primeiros dois anos de vida. Além disso, alta frequência de uso de medicamentos e internação hospitalar demonstram o impacto da doença pulmonar obstrutiva neste grupo etário.
\end{abstract}

DESCRITORES: PREVALÊNCIA; IMPACTO NO ESTADO DE SAÚDE; SIBILÂNCIA; CRIANÇA.

\begin{abstract}
Aims: To identify the prevalence of wheezing and associated factors in children younger than three years of age in a sample of low income region, and to measure other markers associated with severity and risk of asthma in early life. Methods: Parents or legal guardians of infants were interviewed at the primary health center in a low-income area from Porto Alegre, Rio Grande do Sul state, and answered a questionnaire designed to estimate the prevalence of wheezing and evaluate the associated risk factors, called EISL (International Study of Wheezing in Infants). We included patients up to 36 months of age visiting for routine examination or vaccination. Patients brought to emergency room were not included.

Results: The study evaluated a total of 153 children with a mean age of $12.6 \pm 9.5$ months. Of them, $63 \%$ had at least one episode of wheezing reported, which occurred one or more times during the first year of life. The average age of the first episode of wheezing was $4.9 \pm 5.3$ months. Use of $\beta 2$-agonists, oral and inhaled corticosteroids was reported by $58.2 \%, 32.7 \%$ and $19 \%$, respectively. More than $59 \%$ of the patients visited an emergency unit due to wheezing episodes and $13 \%$ were hospitalized at least once in the first years of life because of wheezing.

Conclusions: This study has shown that the prevalence of wheezing on young children is high in a low-income area from Porto Alegre, and the symptoms usually occur during the first two years of life. In addition, high frequency of medication usage and hospitalization demonstrates the impact of obstructive lung disease in this age group.
\end{abstract}

KEY WORDS: PREVALENCE; IMPACT IN THE HEALTH CONDITION; WHEEZING; CHILD. 


\section{INTRODUÇÃO}

Durante os três primeiros anos de vida, várias doenças podem se apresentar clinicamente com quadro de sibilância, sendo este sintoma um motivo frequente de procura por atendimento médico em serviços de urgência. ${ }^{1}$ Crianças menores de dois anos de vida e que manifestam pelo menos três episódios de "chiado", em espaço de seis meses, são denominados "bebês chiadores" ou "lactentes sibilantes". ${ }^{2}$ Várias podem ser as causas de sibilância nessa faixa etária e, embora a asma seja comum, deve ser sempre considerada como diagnóstico de exclusão. ${ }^{1}$ A prevalência da síndrome do lactente sibilante ou de sibilância recorrente é difícil de ser estabelecida, e os estudos que tentaram avaliá-la em crianças menores de um ano mostram índices distintos que oscilam entre $10 \%$ e $42 \% .{ }^{3} \mathrm{~A}$ variação observada entre os estudos pode estar relacionada à falta de padronização dos métodos empregados na identificação desses lactentes, ao tipo de estudo realizado, à definição de sibilância e ao limite da faixa etária estudada. Acredita-se que um terço dos que iniciaram a sibilância antes dos três anos de vida com ela persistirão e, dentre estes, $60 \%$ manifestarão sinais de atopia aos seis anos de idade. ${ }^{4,5}$

As infecções virais, sobretudo pelo vírus respiratório sincicial ou pelo rinovírus, têm sido os principais fatores infecciosos relacionados ao aparecimento de sibilância em crianças, especialmente em não atópicos. ${ }^{6}$ Estima-se que a diferença na prevalência de sibilância entre lactentes de países desenvolvidos e em desenvolvimento seja determinada principalmente por exposições aos fatores de risco ambientais, especialmente aqueles relacionados à condição socioeconômica menos favorecida ${ }^{3}$

O presente estudo visa identificar a prevalência, gravidade e fatores associados à sibilância em crianças com idade inferior a três anos de uma amostra populacional de crianças de baixa renda da cidade de Porto Alegre.

\section{MÉTODOS}

O Centro de Saúde Bom Jesus, situado no distrito leste do município de Porto Alegre, no bairro de mesmo nome, promove atendimento de diversas especialidades, dentre elas a pediatria. A população do bairro é estimada em 28.229 (censo Instituto Brasileiro de Geografia e Estatística 2000). Considerando que a população infantil de 0 a 3 anos de Porto Alegre representa cerca de $6 \%$ da população total (Fundação de Economia e Estatística RS), pode-se estimar que no bairro Bom Jesus o número de crianças até três anos seja de 1.694. Levando em conta um intervalo de confiança com nível de $95 \%$ e uma prevalência estimada em $10 \%$ (asma), seriam necessárias 128 crianças para uma amostra representativa da população infantil do bairro Bom Jesus (http://sampsize.sourceforge.net/ iface/\#prev).

Este estudo foi apreciado e aprovado pelo Comitê de Ética em Pesquisa da Secretaria Municipal de Saúde de Porto Alegre. Os pais ou responsáveis pelas crianças com idade entre zero e 36 meses de vida, que foram levadas para consulta de puericultura, foram entrevistados no próprio centro de saúde, sendo preenchido um questionário padronizado, denominado EISL (Estudo Internacional de Sibilância em Lactentes). Esse questionário, do "Estudio Internacional de Sibilâncias en Lactantes" 7,8 foi desenvolvido com o objetivo de estudar o impacto da sibilância de repetição em lactentes nos seus primeiros 12 meses de vida, determinando sua prevalência e os fatores de risco a ela associados. O protocolo EISL tem como instrumento de aplicação um questionário escrito padronizado, constituído por 45 perguntas sobre características demográficas, sibilância e sintomas respiratórios, consumo de medicações, diagnósticos médicos e possíveis fatores de risco, a ser respondido pelos pais e/ou responsáveis dos lactentes em consultas de rotina. ${ }^{8}$ Neste estudo foram utilizadas somente as 12 perguntas referentes à epidemiologia da sibilância, conforme descrição prévia. ${ }^{9}$ Pacientes trazidos na emergência ou com diagnóstico de asma não foram incluídos no estudo. Visando a privacidade que a referida entrevista requer, os questionários foram entregues e devidamente preenchidos nas salas de atendimento do setor ambulatorial do Centro de Saúde Bom Jesus.

\section{RESULTADOS}

O estudo incluiu um total de 153 lactentes, com idade média de 12,6 $\pm 9,5$ meses (Tabela 1). No grupo entrevistado, $63 \%$ dos lactentes apresentaram chiado ou cansaço no peito, sintoma que apresenta boa correlação com sibilância detectada pela ausculta neste questionário. ${ }^{8}$ As crises ocorreram uma ou mais vezes durante os primeiros anos de vida, sendo a idade

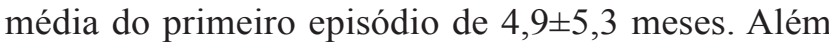
disso, $58,2 \%$ procuraram serviços de emergência por crise de chiado ou cansaço no peito, $47,7 \%$ tinham história de crises com dificuldade respiratória, e 15,7\% necessitaram internação por crise.

Quanto ao uso de medicamentos, 58,2\% utilizaram $\beta 2$-agonistas como salbutamol ou fenoterol, incluindo tanto a nebulização como o uso do spray com espaçador, 
e $32,7 \%$ receberam corticosteroide oral, como prednisolona. Por outro lado, somente $19 \%$ receberam corticosteroide inalado, como beclometasona em spray com espaçador. Mais de $58 \%$ dos pacientes foram à emergência por chiado ou cansaço no peito, e $60 \%$ apresentaram episódio de chiado à noite.

Dentre os entrevistados, $15.6 \%$ tiveram o diagnóstico médico de asma, e $19.6 \%$ o de rinite alérgica. A prevalência de asma na família, em algum parente de primeiro grau, foi de $47,7 \%$ (Tabela 2 ).

Tabela 1. Dados descritivos de 153 crianças com idade até 36 meses, entrevistadas no Centro de Saúde do bairro Bom Jesus, Porto Alegre, RS.

\begin{tabular}{lcc}
\hline \multicolumn{1}{c}{ Variáveis } & Média & $\begin{array}{c}\text { Desvio } \\
\text { padrão }\end{array}$ \\
\hline Idade (meses) & 12,68 & 9,46 \\
Peso de nascimento (gramas) & $3.188,04$ & 673,51 \\
Peso atual (gramas) & $9.144,44$ & $3.363,66$ \\
Estatura ao nascimento (cm) & 48,07 & 4,96 \\
Estatura atual (cm) & 70,75 & 13,04 \\
Idade da primeira crise sibilância (meses) & 5,00 & 5,34 \\
\hline
\end{tabular}

Tabela 2. Resultados do questionário EISL (Estudo Internacional de Sibilância em Lactentes) em 153 crianças com idade até 36 meses, entrevistadas no Centro de Saúde do bairro Bom Jesus, Porto Alegre, RS.

\begin{tabular}{lcc}
\hline \multicolumn{1}{c}{ Variáveis } & N & \% \\
\hline Apresentaram sibilância & 96 & 62,75 \\
Usaram $\beta$ 2-agonistas & 89 & 58,17 \\
Receberam corticosteroide inalatório & 29 & 18,95 \\
Receberam corticosteroide oral & 50 & 32,68 \\
Tinham história de sibilância à noite & 92 & 60,13 \\
Tinham história de crises com dificuldade & 73 & 47,71 \\
respiratória & & \\
Tiveram internação por sibilância & 24 & 15,69 \\
Fizeram visita a emergência por sibilância & 89 & 58,17 \\
Tinham diagnóstico médico de asma & 24 & 15,69 \\
Tinham diagnóstico médico de rinite alérgica & 30 & 19,61 \\
Tinham história familiar de asma & 44 & 28,76 \\
\hline
\end{tabular}

\section{DISCUSSÃO}

A sibilância é motivo frequente de procura por atendimento médico em serviços de urgência, sobretudo quando há recorrência dos episódios. A sibilância de repetição em lactentes é um problema frequente na prática pediátrica. ${ }^{10}$ Entretanto, a maior parte dos estudos tem avaliado a incidência e o impacto da bronquiolite aguda ou da asma em crianças maiores, e o impacto e a prevalência da sibilância recorrente em lactentes tem sido descrito em poucos estudos. ${ }^{2,11} \mathrm{O}$ desconhecimento destes dados é ainda maior em países em desenvolvimento e populações de baixa renda, embora existam alguns relatos limitados ao primeiro ano de vida. ${ }^{12}$

Levantamentos em países da Europa encontraram prevalências de cerca de 45\% (pelo menos um episódio), com alta recorrência dos episódios $(15 \%)^{13}$ Em países da América Latina, as prevalências dos sintomas recorrentes podem ser maiores $(22 \%),{ }^{13}$ determinando elevados gastos e, consequentemente, desfechos mais graves, considerando as limitações do sistema de saúde. As razões para estas possíveis diferenças ainda não estão claras, mas podem ser determinadas principalmente pelas exposições a fatores de risco ambientais, especialmente aqueles relacionados à condição socioeconômica baixa ${ }^{3,12} \mathrm{~A}$ doença respiratória dependeria do grau de exposição da criança a agentes infecciosos e não infecciosos desencadeantes da doença e da susceptibilidade do organismo infantil. Esses determinantes seriam condicionados essencialmente pelo estado de salubridade do meio ambiente e pelo estado nutricional da criança que, consequentemente, estariam ligados à renda familiar. ${ }^{14}$

No presente estudo, realizado com 153 crianças

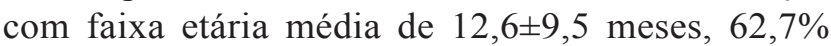
apresentaram sibilância, que durante o primeiro ano de vida ocorreu uma ou mais vezes. A idade média do primeiro episódio de sibilância foi $4,9 \pm 5,3$ meses. Destes, 58,2\% fizeram uso de $\beta 2$ agonistas, $32,7 \%$ utilizaram corticosteroides orais e $18,3 \%$ corticosteroides inalatórios. Estes dados mostram o grande impacto do problema em termos de gastos, mas especialmente em relação à saúde dessas crianças, pois já foi reconhecido que crianças que utilizaram cursos de corticosteroides orais por mais de três episódios podem apresentar osteopenia e risco mais elevado de fraturas mais tarde, na idade escolar e na adolescência. ${ }^{15}$

De 96 crianças que apresentaram sibilância nos primeiros 12 meses de vida, 58,2\% procuraram emergência, e $15,7 \%$ foram internadas por crise de sibilância. Estes dados já demonstram o grande impacto da doença de via aérea nos gastos com saúde pública. Dessa forma, alguma intervenção preventiva, diagnóstica e terapêutica, torna-se essencial. ${ }^{16}$ Entretanto, a etiologia da sibilância no lactente é muito variável. Os fatores que determinam o aparecimento, a evolução e o prognóstico da sibilância em lactentes não estão bem delimitados, porém certamente envolvem imunocompetência do hospedeiro, fatores de risco, fatores predisponentes e a patogenicidade dos agentes 
agressores. ${ }^{1}$ Vários estudos apontam tabagismo passivo, tabagismo na gestação e função pulmonar diminuída ao nascimento, ${ }^{4}$ idade, sexo masculino, má condição socioeconômica, aspiração crônica, atopia e poluição atmosférica como fatores envolvidos no desenvolvimento da síndrome do "bebê chiador", mas na grande maioria, as manifestações clínicas são associadas às infecções virais. ${ }^{17}$ Sabe-se que em muitos casos de asma persistente os sintomas iniciais ocorrem durante os primeiros anos de vida; porém, tal diagnóstico é difícil nessa faixa etária, pois os métodos complementares de investigação, como a função pulmonar, são disponíveis em poucos centros, e o número de potenciais etiologias é grande. ${ }^{15}$

Independente da etiologia, a história familiar de asma (materna, paterna ou em irmãos) eleva significativamente o risco de ocorrência e recorrência de sibilância. Em nosso estudo, 47,3\% das crianças apresentavam história familiar de asma em familiar de primeiro grau, o que pode contribuir para estimar o risco de desenvolverem asma. ${ }^{5,18,19}$ Além disso, outros fatores como prematuridade, baixo peso ao nascimento, irmãos no domicílio, baixo nível socioeconômico e cultural dos pais, também são considerados relevantes para prever riso e recorrência.

Como mencionado anteriormente, a asma da criança comumente inicia no primeiro ano de vida; sendo assim, a identificação de lactentes e préescolares com alto risco de desenvolvimento da doença é uma prioridade em saúde pública ${ }^{19}$ É, portanto, fundamental investigar as crianças com sibilância recorrente, considerando-se o risco de desenvolverem asma, sobretudo quando a sibilância estiver associada a atopia. ${ }^{18}$ Além disso, a intensidade dos sintomas respiratórios durante os dois primeiros anos de vida pode estar fortemente relacionada ao seu prognóstico na vida adulta. ${ }^{8}$

Uma maior atenção à prevalência e melhor compreensão da etiologia da sibilância e diagnóstico da asma em lactentes e pré-escolares podem trazer novas estratégias que visem diminuir não somente a morbidade nesses pacientes, mas também os excessivos gastos com a doença. O estudo ISAAC (International Study of Asthma and Allergies in Childhood) foi idealizado em 1991, na Austrália, para verificar a prevalência de asma e doenças alérgicas em crianças e adolescentes de todo o mundo e compará-las de modo mais fidedigno ${ }^{20} \mathrm{O}$ protocolo ISAAC propôs um método único de pesquisa e permitiu maximizar o valor dos estudos epidemiológicos em asma e doenças alérgicas. Esse protocolo teve como pontos específicos: a) descrever a prevalência e a gravidade da asma, rinite e eczema em crianças e adolescentes em diferentes centros e realizar comparações de países e entre eles; b) obter medidas basais para assessorar futuras tendências na prevalência e gravidade dessas doenças; c) fornecer estruturas para estudos etiológicos posteriores. ${ }^{20}$

O estudo ISAAC demonstrou uma elevada prevalência de asma no Brasil. Sete centros (Recife, Salvador, Uberlândia, Itabira, São Paulo, Curitiba e Porto Alegre) participaram do estudo. Na fase I do estudo, 13.604 escolares (6 a 7 anos) e 20.554 adolescentes (13 a 14 anos) foram avaliados. A prevalência de asma ativa (sibilância nos últimos 12 meses) nos escolares atingiu 27,2\% em Porto Alegre, enquanto que entre os adolescentes, as taxas de prevalência variaram entre 9,6\% (em Itabira) e $27,1 \%$ (em Salvador). Por outro lado, os dados sobre sibilância nos primeiros anos de vida são escassos e dificultam a comparação entre países. ${ }^{21}$ Já o protocolo EISL é um projeto mais recente e ainda foi estudado em poucos locais, especialmente quando comparado ao projeto ISAAC. Dessa forma, considera-se que a sibilância nos primeiros anos de vida realmente parece necessitar de mais investigação, tanto no que refere a prevalência e etiologia, como também ao manejo dessa situação.

A prevalência elevada $(>60 \%)$ nesta amostra chama atenção e confirma a alta prevalência descrita em outros trabalhos que estimaram a prevalência de sibilância somente durante o primeiro ano de vida. ${ }^{12,22}$ Estes valores extremamente elevados também precisam ser avaliados considerando-se o efeito cumulativo. Ou seja, como o tempo avaliado foi maior neste estudo (3 anos), a chance de o indivíduo apresentar um episódio também aumenta. De qualquer forma, este número, que mostra que mais da metade das crianças apresentaram pelo menos uma crise, merece atenção quando decidimos sobre prioridades em saúde pública.

Segundo dados da coorte de Tucson (Arizona, Estados Unidos), até os seis anos de idade, aproximadamente $50 \%$ das crianças apresentam pelo menos um episódio de sibilância, ${ }^{4}$ a maioria causada ou desencadeada por infecções virais. Entretanto, a gravidade e as características clínicas podem variar significativamente. As crianças infectadas por um mesmo vírus podem apresentar desde um quadro de sibilância leve sem dificuldade respiratória até crises graves com necessidade de internação, seguidas por sintomas recorrentes ou persistentes. ${ }^{23}$ Essa grande variação pode ser influenciada por fatores genéticos e imunológicos.

Considerando sua importância para saúde pública, a realização de pesquisas de prevalência e etiologia nesta área são de extrema importância para estimar o 
impacto e planejar a atenção destinada a populações de baixa renda. A partir dos dados de prevalência de sibilância nesta população, medidas para melhorar o diagnóstico e tratamento, assim como ações preventivas poderão ser construídas ou intensificadas no intuito de reduzir o impacto de sibilância, bem como reduzir os excessivos gastos com este problema de saúde.

\section{REFERENCIAS}

1. Eigen H. Differential diagnosis and treatment of wheezing and asthma in young children. Clin Pediatr (Phila). 2008 Oct; 47(8):735-43.

2. Chong Neto HJ, Rosário NA, Grasselli EA, Silva FC, Bojarski Lde F, Rosário CS, Rosário BA, Chong FH. Recurrent wheezing in infants: epidemiological changes. J Pediatr (Rio J). 2011 Nov-Dec;87(6):547-50.

3. Garcia-Marcos L, Mallol J, Solé D, Brand PL; EISL Study Group. International study of wheezing in infants: risk factors in affluent and non-affluent countries during the first year of life. Pediatr Allergy Immunol. 2010 Aug;21(5): 878-88.

4. Martinez FD, Wright AL, Taussig LM, Holberg CJ, Halonen M, Morgan WJ. Asthma and wheezing in the first six years of life. The Group Health Medical Associates. N Engl J Med. 1995 Jan 19;332(3):133-8.

5. Castro-Rodriguez JA, Cifuentes L, Rodriguez-Martinez CE. The asthma predictive index remains a useful tool to predict asthma in young children with recurrent wheeze in clinical practice. J Allergy Clin Immunol. 2011 Apr;127(4):1082-3.

6. Chong Neto HJ, Rosário NA; Grupo EISL Curitiba (Estudio Internacional de Sibilancias en Lactantes). Risk factors for wheezing in the first year of life. J Pediatr (Rio J). 2008 Nov-Dec;84(6):495-502.

7. Bianca AC, Wandalsen GF, Miyagi K, Camargo L, Cezarin D, Mallol J, Solé D. International Study of Wheezing in Infants (EISL): validation of written questionnaire for children aged below 3 years. J Investig Allergol Clin Immunol. 2009;19(1):35-42.

8. Mallol J, García-Marcos L, Aguirre V, Martinez-Torres A, Perez-Fernández V, Gallardo A, Calvo M, Rosario Filho N, Rocha W, Fischer G, Baeza-Bacab M, Chiarella P, Pinto R, Barria C. The International Study of Wheezing in Infants: questionnaire validation. Int Arch Allergy Immunol. 2007;144(1):44-50.

9. Chong Neto HJ, Rosário NA, Solé D, Mallol J. Prevalence of recurrent wheezing in infants. J Pediatr (Rio J). 2007 July-Aug;83(4):357-62.

10. Cavalcanti Dela Bianca AC, Wandalsen G, Prestes E, Lamenha M, Bessa O, Chong Neto H, Medeiros D, Sarinho EC, Rosario Filho NA, Mallol J, Solé D. Treatment of wheezing in Brazilian infants in the first year of life. Pediatr Allergy Immunol. 2013 Oct 7.

11. Dela Bianca AC, Wandalsen GF, Mallol J, Solé D. Prevalence and severity of wheezing in the first year of life. J Bras Pneumol. 2010 Jul-Aug;36(4):402-9.

12. Mallol J, Andrade R, Auger F, Rodríguez J, Alvarado R, Figueroa $\mathrm{L}$. Wheezing during the first year of life in infants from low-income population: a descriptive study. Allergol Immunopathol (Madr) 2005;33(5):257-63.

13. Mallol J, García-Marcos L, Solé D, Brand P; EISL Study Group. International prevalence of recurrent wheezing during the first year of life: variability, treatment patterns and use of health resources. Thorax. 2010 Nov;65(11):1004-9.

14. Moraes LS, Takano OA, Mallol J, Solé D. Risk factors associated with wheezing in infants. J Pediatr (Rio J). 2013 Nov-Dec;89(6):559-66.

15. Gillissen A, Wirtz H, Hoheisel G. [New perspectives in GINA Asthma Guideline 2006]. Med Klin (Munich). 2007 May 15;102(5):399-403.

16. Covar RA, Spahn JD. Treating the wheezing infant. Pediatr Clin North Am. 2003 Jun;50(3):631-54.

17. Tian M, Zhao DY, Wen GY, Shi SY. [The correlation factor about respiratory syncytial virus bronchiolitis and postbronchiolitis wheezing in infant]. Zhonghua Shi Yan $\mathrm{He}$ Lin Chuang Bing Du Xue Za Zhi. 2009 Oct;23(5):371-4.

18. Castro-Rodriguez JA. The Asthma Predictive Index: early diagnosis of asthma. Curr Opin Allergy Clin Immunol. 2011 Jun;11(3):157-61.

19. Chang TS, Lemanske RF Jr, Guilbert TW, Gern JE, Coen $\mathrm{MH}$, Evans MD, Gangnon RE, Page CD, Jackson DJ. Evaluation of the Modified Asthma Predictive Index in High-Risk Preschool Children. J Allergy Clin Immunol Pract. 2013 Mar 1;1(2).

20. Lis G, Breborowicz A, Cichocka-Jarosz E, Swiatły A, Głodzik I, Gazurek D, Sobkowiak P, Alkiewicz J, Pietrzyk JJ; International Study of Asthma and Allergies in Childhood. [Increasing prevalence of asthma in school children-ISAAC study (International Study of Asthma and Allergies in Children)]. Pneumonol Alergol Pol. 2003;71 (7-8):336-43

21. Solé D, Yamada E, Vana AT, Costa-Carvalho BT, Naspitz CK. Prevalence of asthma and related symptoms in schoolage children in Sao Paulo, Brazil--International Study of Asthma and Allergies in Children (ISAAC). Asthma. 1999;36(2):205-12.

22. Lima JA, Fischer GB, Sarria EE, Mattiello R, Solé D. Prevalence of and risk factors for wheezing in the first year of life. J Bras Pneumol. 2010 Sept-Oct;36(5):525-31.

23. Rusconi F, Galassi C, Bellasio M, Piffer S, Lombardi E, Bonci E, Armenio L; Gruppo Collaborativo SIDRIA-2. [Risk factors in the pre-, perinatal and early life (first year) for wheezing in young children]. Epidemiol Prev. 2005 MarApr;29(2 Suppl):47-51. 